Rev. Est. de Políticas Públicas, 5(2): julio 2019 - noviembre 2019, 42-57

http://dx.doi.org/10.5354/0719-6296.2019.55356

ISSN edición web: 0719-6296

(C)Copyright 2019: Universidad de Chile, Santiago (Chile)

\title{
Entre la economía moral del valor justo y la economía política del precio de mercado. El desarrollo de capacidades estatales y la regulación de las relaciones sociales de mercado a partir de las políticas de control de precio
}

\author{
Juan Pablo Quiroga
}

FLACSO, Argentina

\begin{abstract}
Resumen
Las políticas de control de precios y regulación de acceso a los alimentos han sido poco estudiadas en término de las relaciones que las vinculan con el Estado, sus agencias, y su capacidad de intervenir en el tejido y dinámica social. En este sentido, se presentan los resultados de comparar diversos programas de control de precio, durante el bienio 2014-2016 en Egipto, Argentina, Venezuela y Estados Unidos. Cuatro países que persiguiendo objetivos similares, lo hicieron bajo modalidades y diseños diferentes: subvención a productos consumidos por sectores sociales vulnerados (Egipto); precios acordados para una cantidad limitada de productos (Argentina); precios congelados para toda la economía (Venezuela); y transferencias condicionadas, en Estados Unidos. Sostendremos que en mercados de transiciones incompletas entre economías morales de provisión y economías políticas de mercado, las políticas de control de precios son parte integral del pacto social. Un argumento que hace de las capacidades estatales un elemento clave de análisis y busca reconocer que, en algún punto, su análisis no sólo debe ponerse en relación con las estructuras de mercado, sino que es su punto de partida necesario.
\end{abstract}

Palabras clave: Capacidades Estatales, Sistema de Precios, Control de Precios, Mercado.

Between the moral economy of fair value and the political economy of the market price

\begin{abstract}
Both the price control policies and those that aim at regulating food access have been little studied in terms of their intimate relations with the State, its agencies, and its capacity to intervene in the fabric and dynamics of society. In this sense, this paper condenses results of comparing various price control and food access programs, during the 2014-2016 biennium in Egypt, Argentina, Venezuela and the United States. Four countries that, pursuing similar objectives, did so under four different designs: subsidies for products consumed by low-income segments of society (Egypt); agreed-prices for a limited quantity of products (Argentina); frozen prices for the whole economy (Venezuela); and conditional transfers, in the United States. We will argue that in markets of incomplete transitions between moral provision economies and political market economies, price control policies are an integral part of the social pact. An argument that makes state capacities a key element of analysis and forces us to recognize that at some point, its analysis should not only be related to market structures, but is its necessary starting point.
\end{abstract}

Keywords: State Capacities, Price System, Price Control, Market.

*Dirección de correspondencia [Correspondence address]:

Juan Pablo Quiroga, FLACSO, Argentina

E-mail: jq84@georgetown.edu

Fecha recepción: 10 de septiembre del 2019

Fecha aceptación: 25 de octubre del 2019 


\section{Introduccion: el debate en el tiem- po}

Las políticas de control de precios se remontarían en su origen al inicio mismo de las sociedades con gobierno centralizado, a partir de cierta noción del valor "justo" de los bienes vinculados a la subsistencia. Incluso, algunos autores han llegado a señalar que el intento por reglamentar el acceso a los mismos fue codificado, por primera vez, en el Código Hammurabi a partir de un rígido sistema de equivalencias entre salarios y precios $^{1}$ (Quiroga, 2015).

Sin embargo, los mismos no serían objeto de análisis sistemático hasta el siglo XX. De hecho, como hemos indicado en otro artículo (Quiroga, 2015), los primeros abordajes serían realizados en primera persona por funcionarios a cargo de experiencias de control: Taussig, 1919; Galbraith, 1941, 1946; G. y Neeb, 1974; y Clinard,1969 .

En este sentido, pueden distinguirse analíticamente tres etapas del debate sobre las políticas de control de precios: (a) durante el período de guerras, en sentido amplio, comprendiendo la Primera Guerra Mundial y la Segunda Guerra Mundial, hasta la guerra de Corea; (b) el debate de posguerra y el período de discusión sobre el alcance de los denominados "Regímenes de Bienestar" en los países centrales $^{2}$; y (c) la redefinición de los roles y alcances de los mismos en mencionados países y de los sistemas de protección social en los países en desarrollo ${ }^{3}$ (Quiroga, 2015).

Ahora bien, en líneas generales existe cierto consenso en la literatura de época sobre la necesidad de los controles de precios durante el período comprendido por la primera etapa (Quiroga, 2015). No

\footnotetext{
"Si un hombre contrata a un trabajador del campo, deberá darle ocho GUR de trigo por año. 258. Si un hombre contrata a un pastor, deberá darle seis GUR de trigo por año. (...) 273. Si un hombre contrata a un trabajador, desde el inicio del año hasta el quinto mes, le deberá pagara seis granos de plata por día. (Schuettinger y Butler, 1978)

2 Preferimos hablar de "debates sobre el alcance" en la intervención de los regímenes de bienestar, para el caso de los países centrales, en vistas a que la idea sobre el "desmantelamiento", "crisis" o "debilitamiento" del Estado de Bienestar es discutida por varios autores (Pierson,2001).

${ }^{3} \mathrm{~A}$ los efectos del presente, mantendremos para el caso de los denominados países centrales, la referencia a "Régimen de Bienestar" en los términos propuestos por de EspingAndersen (1993), mientras que - por las polémicas suscitadas en la aplicación de mencionado concepto para el |contexto de los países en desarrollo en general y América Latina en particular (Mesa-Lago, 2004; Filgueira, 2001 y 2014; Martinez Franzoni, 2008; Ocampo, 2008, por mencionar algunas), nos referiremos a "sistemas de protección social".
}

sólo por la urgencia de administrar el stock de productos disponibles frente al faltante provocado por la asistencia a las fuerzas en guerra, sino también como modalidad de organizar la producción y racionalizar el consumo de bienes necesarios en el frente de combate, como acero, caucho, nafta, entre otros (Clinard,1969).

Sin embargo, a diferencia de esta legitimidad de excepción sobre la que se fundaron los controles de guerra (marcada incluso, a nivel de la opinión pública, por ciertos componentes patrióticos), durante tiempos de paz distintos gobiernos debieron intervenir el mercado de bienes de consumo, en búsqueda de limitar el aumento de precios. En esta segunda etapa, la discusión sobre las políticas de control de precios fue coextensiva a los debates sobre el alcance de la intervención estatal en el marco de los denominados Regímenes de Bienestar en los países centrales, o bien de los sistemas de protección social emergentes en los países en desarrollo (Quiroga, 2015).

En una tercera etapa, en cambio, las discusiones sobre las políticas de control de precio evidenciarían, como hemos señalado en otra fuente (Quiroga, 2015), un giro drástico que definen su estatus al día de hoy. En primer lugar, los controles extensivos quedarán desacreditados de la mano de la búsqueda tanto por focalizar como por subsidiar la demanda. De esta forma, en lugar de controles comprensivos a lo largo de la economía, los mismos se limitarían, sobre todo en los países centrales, a medicamentos y transporte. Incluso, en lo relativo a los bienes de consumo, los programas buscarían progresivamente no cubrir al total de la población sino focalizarse en segmentos identificados como los más necesitados a tales fines..

Asimismo, los controles perderán especificidad propia en el debate para quedar subsumidos bajo la órbita del campo emergente de la seguridad alimentaria (Quiroga, 2015), definida en el marco de la Cumbre Mundial sobre la Alimentación de 1996 a partir de tres elementos centrales: la disponibilidad de alimentos, la accesibilidad a los mismos, su utilización y la estabilidad misma de todo el proceso. La problemática de los precios, entraría - de esta formaentre la serie de preocupaciones tendientes a garantizar el acceso a los alimentos, en conjunto con otras como: la existencia de fuentes propias de alimentación, como stocks propios, la producción casera, los circuitos de trueque, regalo, préstamos, remesas y/o asistencia alimentaria, entre otros. 


\section{Las propiedades invariantes del debate}

De la revisión bibliográfica sobre las políticas de control de precios se evidencia no sólo una pérdida progresiva de autonomía de las políticas de control de precios, sino una serie de invariantes en el devenir social de su debate (Quiroga, 2017b).

En primer lugar, se hace manifiesto un conjunto de referencias semánticas y problemáticas propias y recurrentes (Quiroga, 2015), entre ellas: las preocupaciones por el rol y el tamaño de la burocracia (Taussig, 1919; G. y Neeb, 1974; Clinard, 1969; Rockoff, 1984; Galbraith, 1941 y 1946); la eficacia (potencial) de corto plazo y la ineficacia de mediano-largo de los controles (Rockoff, 1984; G. y Neeb, 1974; Bienen y Gersovitz, 1986, Fiel, 1990); la evasión y la constitución de mercados negros (Clinard, 1969); la eventual violencia emergente tras el desmantelamiento de los controles o subsidios a los bienes de consumo (Bienen y Gersovitz, 1986); su potencial impacto en la coalición de gobierno (Agenor y Asilis, 1997); los niveles de entrega o incluso eventuales quiebres en las cadenas de abastecimiento (G. y Neeb, 1974; Clinard, 1969); los incentivos/distorsiones en la distribución de recursos, como fruto de la alteración del sistema de precios (Galbraith, 1941, 1946, 1951, 2001; Friedman, 1990; Colander, 1984; Dunn y Pressman, 2005); la búsqueda de alternativas focalizadas con miras a optimizar el uso de recursos a partir de limitar el denominado free-riding (Adams, s/f; Alderman,2002; Gutner,2002); e incluso su devenir histórico como respuesta al alza de precios (Schuettinger y Butler, 1978).

Una segunda invariante, estaría dada por la estabilidad (relativa) de las formas que asumieron los controles en el tiempo (Quiroga, 2015). De hecho, de la literatura se desprende una tipología de cinco hechos estilizados en materia de controles de precios directos. En primer lugar, los "precios vigilados" en donde la atención se centra en las cadenas de comercialización, las cuales deben avisar a la Secretaría de Comercio sobre eventuales cambios en los precios. En segundo término, el denominado "acuerdo de precio", por el cual empresas comercializadoras y proveedores pactan los valores de un set de productos. Una tercera opción, en cambio, es referida, con frecuencia, como "precios controlados" y consiste la necesidad por parte de productores y comercializadores de obtener aprobación de la Secretaría de Comercio para realizar cambios de precio. En cuarto lugar, tendríamos los "precios máximos" por los cuales la autoridad competente fija un límite superior para determinados productos; mientras que, por último, estaría el "congelamiento generalizado" por un tiempo determinado, en los cuales el gobierno restringe de forma absoluta los cambios en materia de precios (Quiroga, 2015).

A esta estabilidad relativa de las formas de controles de precios, se le suman ciertas políticas indirectas de control de precios (Quiroga, 2015). Entre ellas, las más recurrentes son (a) subsidios generales al consumo; (b) sistemas de auto-focalización [selftargeting], como el descripto más arriba para el caso de Egipto; (c) exenciones impositivas; (d) establecimiento de cuotas y mecanismos de racionalización del tipo first-come-first-served y (e) identificación de dinámicas y canales de consumo diferenciados por segmentos sociales, con miras a su identificación y focalización (Alderman,2002:4-11).

Por último, existe una tercera propiedad invariante en el análisis, de suma importancia a los efectos de nuestra investigación, la cual sólo se hace evidente por su omisión en el debate: la discusión sobre las capacidades (presuntas) del Estado (Quiroga, 2015). Toda la discusión sobre las políticas de control de precios da por hecho, como constante, la existencia de capacidades para su realización. Las diferencias, en cambio, sólo radican en el acento valorativo en que se enmarque la mencionada intervención, pero nunca son puestas en duda en su existencia. Es decir, o bien el Estado tiene capacidades para controlar los precios, o bien para distorsionar el sistema de mercado, pero bajo ningún punto no tiene capacidades.

\section{Alcance, objetivos y metodología}

Es a partir de la identificación de estas invariantes que la investigación doctoral buscaba analizar las variaciones en la capacidad estatal de las agencias implicadas en el control de precios en Argentina, Venezuela, Egipto y Estados Unidos durante el bienio 2014-2016, como fruto del diseño y ejecución de políticas diferentes, partiendo del supuesto que distintos diseños institucionales generan distintas capacidades de acción.

Es decir, dentro del campo delimitado por la problemática general de la(s) capacidad(es) estatal(es), el diseño teórico-metodológico que nos proponíamos limitaba el conjunto posible de fenómenos a estudiar dentro de la temática en cuestión, a partir de operacionalizar la propiedad conceptual "capacidades estatales" como medida de las dotaciones materiales, 
financieras, legales, organizacionales y humanas al interior de cuatro componentes (Bertranou,2013). A saber: (a) los vínculos con actores no-estatales; (b) la legitimidad del sujeto estatal; (c) la organización y medios de acción: las características de los arreglos institucionales y la estructura burocrática; y (d) el capital de acción interinstitucional. Asimismo, la unidad de análisis quedaría limitada a las alternativas institucionales desarrolladas para el control de precios en cuatro países, Argentina, Venezuela, Estados Unidos y Egipto, entre los años 2014 y 2016.

De esta forma, nuestra estrategia tenía por objeto trabajar con casos de un mismo tipo ("políticas de control de precio") pero ligeramente distintos (modalidades de subsidio a la oferta, subsidio a la demanda, acuerdo de precios sobre una canasta limitada de productos y congelamiento total de los precios de una economía).

Por otro lado, nuestra metodología de trabajo, en lo relativo a la recolección de datos, contemplaba tanto fuentes primarias, como la realización de encuestas, entrevistas en profundidad a referentes de las principales cadenas de supermercados, cámaras del sector y funcionarios de gobierno; como secundarias: artículos periodísticos, índices de consumo, legislación, entre otros.

En esta línea, la estrategia metodológica que mejor se ajustaba al diseño de investigación que nos proponíamos era el estudio de casos múltiples. Sobre todo, porque al intentar dar cuenta de modalidades institucionales diferentes para controlar los precios y facilitar el acceso a bienes de consumo masivo en países con realidades institucionales tan diferentes, la reconstrucción - a partir de la descripción detallada de las relaciones entre los componentes de la capacidad estatal y otras variables como régimen político, estructura burocrática, estructura de mercado, entre otras- revisten de un valor central. En este punto, el foco de la investigación -aun cuando mantiene cierto interés en comparar los resultados de cada caso entre sí- pone un énfasis mayor en dar cuenta el fenómeno de interés, su contexto y la relación dinámica entre ambos, a partir del desarrollo de una estrategia metodológica comprensiva que produzca un gran número de variables potenciales relevantes.

Asimismo, la estrategia de estudio de casos múltiple nos permitía un doble límite en cuanto al alcance de la investigación. Nos ofrecía un freno a los potenciales excesos en intensidad descriptiva, a la vez que nos limitaría en cuanto a su contrario: las pretensio- nes de extensividad, propias de los estudios comparativos. Este doble límite, en profundidad y extensión, nos posiciona un rango de acción intermedio en donde sin renunciar ni sacrificar la comprensión-encontexto de cada uno de los casos, ni concentrarnos exclusivamente en rasgos generalizantes que posibiliten la comparación (como ocurre con ciertas estrategias exclusivamente comparativas, al situarse en un nivel superior de generalización o tipificación lógica), podríamos realizar ciertas comparaciones de alcance limitado.

\section{Los casos y su particularidad de forma}

Como adelantábamos en el apartado anterior, la elección de cada uno de los casos de estudio estaba hecha de forma tal que, manteniendo invariante su objeto, nos posibilitase concentrarnos en la diversidad de entramados institucionales que le da forma y sustento. En este sentido, a continuación, ofrecemos una breve descripción de los principales rasgos de los mismos.

\subsection{Argentina}

En enero de 2014 nace en Argentina el programa de Precios Cuidados. Un acuerdo entre la Secretaría de Comercio (dependiente del Ministerio de Economía de la Nación), los supermercados, los distribuidores y sus principales proveedores para la administración de los precios de un set (inicial) de 194 productos durante todo 2014, con renegociaciones trimestrales entre las partes.

El mismo tendría tres objetivos declarados: (a) asegurar condiciones de competitividad en la economía, (b) "cuidar el bolsillo" de los argentinos, y (c) que cada consumidor ejerza su derecho de elegir informado. El diseño del programa buscaba, de esta forma, establecer y dar visibilidad a una canasta de productos con "precios de referencia" que contemplen "márgenes razonables" de rentabilidad. Es decir, se trataría de una respuesta pública a un presunto problema de ambición privada y márgenes de ganancia, en distintos puntos de la estructura de costos, tanto de los sectores productivos, como de distribución.

En su etapa inicial, el mismo buscó administrar el precio de 194 productos, producidos por 75 empresas proveedoras y distribuidos por 10 cadenas de supermercados de todo el país. Sin embargo, a lo largo del año 2014 fue incorporando nuevos ítems, como 
fruto de las revisiones trimestrales, con aumentos de precio, en los períodos mencionados, del orden del $3 \%$ en promedio.

Por otro lado, en relación a los resultados del programa, en tanto que "cambios observables en las condiciones de vida de la población" (Bertranou,2013:18), según datos oficiales la participación de los productos de Precios Cuidados en la facturación de los supermercados ascendió del $5 \%$ al $20 \%$. Un dato que las fuentes privadas, consultadas en una primera versión del presente estudio (Quiroga, 2015), ratifican y ubican -según la cadena en cuestión- entre el $14 \%$ y el $18 \%$.

En cuanto a los productos que forman parte de la canasta del programa, los mismos evidenciaron en dos meses un aumento del $700 \%$ promedio en su demanda, según los empresarios entrevistados (Quiroga, 2015). Conforme lo mencionado por los mismos, tomando el mes de abril de 2014 y comparándolo con el mismo mes de 2013 (cuando no existía el programa), los productos de Precios Cuidados verificaron un incremento en su facturación del $159 \%$. De hecho, según una de las cadenas, comparando la demanda en unidades de la canasta en febrero de 2015 contra febrero de 2013 (cuando no existía el programa), la venta de los productos en cuestión habría subido un $21 \%$ (Quiroga,2015).

\subsection{Venezuela}

En Venezuela, por otro lado, se presentaría al mismo tiempo (el 22 de enero de 2014) el programa de Precios Justos (Quiroga, 2015). El mismo supondría una batería legal más compleja y densa que Precios Cuidados a los efectos de asegurar el rol del gobierno de (a) fijar precios máximos de todos los bienes disponibles en el mercado de consumo, a partir de establecer un techo del $30 \%$ a los márgenes de ganancia; y (b) fiscalizar su implementación, al punto de poder expropiar mercadería y venderla de forma directa al público. De hecho, desde el 23 de enero de 2014, en sólo dos años se sancionó: una ley ("Ley Orgánica de Precios Justos"); una reforma a la misma para incorporar más artículos; y diez resoluciones, las cuales además de operacionalizar la ley marco, dictan adecuaciones sobre faltantes, como leche, jabón, arroz, maíz, entre otros (Quiroga,2015).

El Gobierno venezolano buscaba evitar, de esta forma, que üsurerosz . especuladores"presionen sobre el precio al consumidor, en un contexto marcado por una inflación del $56 \%$, según cifras del Banco Cen- tral de Venezuela.

Por otro lado, se creaba una nueva agencia, la Superintendencia Nacional para la Defensa de los Derechos Socieoeconómicos (Sundde), con la misión de fijar precios máximos a lo largo de toda la cadena de valor: desde la producción hasta la importación, pasando por la distribución y el consumo. Asimismo, se establecía que todo comerciante debía inscribirse en el Registro Único de Personas que Desarrollan Actividades Económicas (Rupdae) para poder ejercer la actividad.

El modelo venezolano, con el objetivo de fijar "criterios justos de intercambio para tener una determinación de porcentajes de ganancia razonables, además de salvaguardar los derechos de los trabajadores y proteger al pueblo contra el boicot económico y la usura", supondría no sólo un control de toda la actividad comercial del país, sino también de la movilización de todo el apartado estatal para su control.

\subsection{Egipto}

En Egipto, en cambio, en 2014 tendría lugar una serie de modificaciones al sistema de subsidios a los alimentos, vigentes desde la independencia misma del país, con miras a reducir el gasto público sin eliminar los programas, dado que su desmantelamiento podía derivar en violencia (Bienen and Gersovitz, 1986 y van Wijnberger,1992) o en costos electorales para la coalición de gobierno (Agenor y Asilis, 1997), algo que ya había ocurrido en el pasado.

El sistema original -vigente desde la Segunda Guerra Mundial- implicaba el subsidio a bienes de consumo de alto valor calórico y bajo valor nutricional, como pan, trigo, arroz, azúcar y aceite de cocina, pero -según varios autores- existían grandes deficiencias. De hecho, progresivamente, no sin intentos fallidos ni violencia, se intentarían realizar cambios en los criterios de elegibilidad, en el número total de beneficiados y el precio de las tarjetas de racionamiento, así como en la producción y distribución del pan baladi (el principal componente del sistema de subsidios, disponible para todos) ${ }^{4}$ como intento por controlar el gasto público.

\footnotetext{
${ }^{4}$ Existen tres grades variedades de pan en la dieta egipcia: el pan baladi, fino y shami. Los mismos se diferencian por la tasa de extracción, es decir: por la cantidad de harina producida a partir de una libra de grano. Cuanto mayor es la tasa, más "rústica" es la textura. En el caso del pan baladi, consumido preferencialmente por sectores de bajos ingresos, la tasa de extracción es del $82 \%$, mientras que la del fino es $76 \%$ y la del shami $72 \%$.
} 
Para junio de 2013 el gobierno gastaba más del $10 \%$ del PBI en subsidios, sin que estos se focalizaran eficientemente en la población beneficiaria. Eran recurrentes los informes de agencias multilaterales en relación a los desvíos del beneficio de su población objetivo, siendo usados como alimento para animales o revendidos como insumos al mercado negro para la producción de otros productos. Según datos del Banco Mundial, por ejemplo, de eliminarse estas "filtraciones" se ahorraría el $73 \%$ de los costos del subsidio (Nawar,2014:91).

En este sentido, mientras en la década de 1980 y 1990 los subsidios a los alimentos decrecieron, tanto como porcentaje del PBI como del gasto de gobierno, la tendencia parecía revertirse (marginalmente) a partir de los años 2000. Sobre todo, como producto del ajuste del sistema para compensar la devaluación de la moneda y el incremento del precio internacional de los alimentos.

Para el año fiscal 2011-2012, los subsidios alcanzarían un valor total de $\$ 18.000$ millones de dólares, presionando sobre las cuentas públicas pero -a su vez- ocupando un lugar central en la política de largo plazo del Estado con miras a promover la equidad social y la estabilidad política.

De esta forma, el sistema auto-focalizado de subsidios al consumo de "bienes inferiores", sería complementado, en febrero de 2014, con una serie de cambios que buscarían controlar problemas de focalización y la formación de mercados negros. Los subsidios al pan baladi dejarían de ser universales: pasarían a comprarse con las tarjetas electrónicas y el acceso a las mismas estaría restringido, a la vez que cada beneficiario recibía una cuota de 150 piezas al mes, con un límite de 40 por día y un mínimo de 5 .

Por otro lado, se dejaría de subsidiar la harina que se distribuía en las panaderías, para que las mismas la obtengan a precio de mercado de los molinos y vendan el pan al precio fijado. El gobierno, de esta forma, sólo subsidiaría la diferencia entre el costo real (incluido el margen) y el precio de venta al público, sin necesidad de controlar toda la cadena de comercialización. Así, se buscaba controlar la fuga de producto al mercado negro, a la vez que las panaderías se convertirían en competidores de mercado con nuevos incentivos para mejorar su eficiencia y productividad.

A su vez, se limitaría el tamaño del beneficio a un máximo de 5 rodajas por individuo por día o $10 \mathrm{~kg}$ de harina subsidiada por mes y se introduciría un sistema de puntos que premiaba el racionamiento. De esta forma, por cada rodaja de pan no consumida, el sistema les daba 10 piastras que podían usarse para la compra de otros productos dentro del sistema de racionamiento.

Por último, se establecería un sistema electrónico de control del consumo efectivo de los beneficiarios y las transacciones financieras de las panaderías. Así, todas las panaderías debían tener un posnet para la venta del pan y los tenedores de tarjetas solo tenían que poner su número de identificación en el lector para retirar su ración.

\subsection{Estados Unidos}

El Programa de Asistencia Nutricional Suplementaria (SNAP, por sus siglas en inglés) es uno de los programas más grandes, vigentes al día de hoy, en Estados Unidos en lo relativo a la asistencia a familias de bajos ingresos. Incluso, es señalado como la piedra angular del sistema de contención en materia de nutrición (Rosenbaum, 2013) y la principal política de lucha contra el hambre de ese país.

Inicialmente empezó sobre el final de la Gran Depresión, para finalizar en 1943 a la luz del boom económico de la posguerra. Luego se formalizaría en un programa piloto en 1964 impulsado por la administración Kennedy, con un objetivo doble: (a) alcanzar un mejor y más eficiente uso de la abundancia de alimentos; y (b) elevar los niveles de nutrición. Finalmente, sería la administración de Johnson la que lo institucionalizaría formalmente como un programa.

Al día de hoy, se trata de un programa de transferencia condicionada, por el cual familias de tres miembros, con un ingreso menor o igual a $\$ 2.177$, cuyos bienes sean menores o iguales a $\$ 2.250$ (o $\$ 3.250$ para un hogar con adultos mayores o inválidos), reciben un beneficio promedio de $\$ 127$ al mes. Un equivalente a $\$ 4,23$ al día o $\$ 1,41$ por comida en 2015 (CBPP, 2016). Por otro lado, casi todos los desempleados sin hijos pueden ser beneficiarios por tres meses, a condición que trabajen un mínimo de 20 horas a la semana o participen de un programa de entrenamiento en empleo, en cuyo caso el plazo de beneficio puede extenderse (Isaacs, 2008). Solo en 2015, el programa brindó asistencia a 45 millones de beneficiarios en 23 millones de unidades domésticas de bajos ingresos, de las cuales el $70 \%$ eran familias con hijos y más del $25 \%$ correspondían a hogares con adultos mayores o personas con alguna 
discapacidad (CBPP, 2016).

Los beneficios son distribuidos a través de una tarjeta electrónica, la cual puede ser usada sólo en negocios autorizados. Al día de hoy, la cantidad de tiendas alcanzadas asciende a 261.000, $80 \%$ de los cuales son supermercados o hipermercados (CBPP, 2016).

No obstante, existen ciertas particularidades en su diseño que configuran su condición de excepción como objeto de estudio. Entre ellas, se destaca el hecho que el monto del beneficio puede variar mes a mes. Es decir, depende de una fórmula que busca identificar los hogares de mayor necesidad. En este sentido, las unidades domésticas más vulnerables, reciben mayores beneficios. La fórmula supone que las familias beneficiarias deberían gastar $30 \%$ de su ingreso neto en comida, por lo que el programa prevé compensar la diferencia entre el $30 \%$ de su ingreso y el costo del Plan de Alimentación Económica [thrifty food plan], una dieta de bajo costo pero nutricionalmente adecuada, establecida por el Departamento de Agricultura (USDA, 2016).

Por último, existe cierto consenso en la literatura sobre la capacidad del programa de responder efectivamente a contextos de depresión económica. En esta línea, entre 2007 y 2011 el número total de beneficiarios se incrementó, mientras que a partir de 2012 comenzó una disminución progresiva, como fruto de la recuperación económica (Rosenbaum, 2013).

En términos comparativos, el interés diferencial de cada propuesta, radica en que mientras Precios Cuidados supondrá un ejercicio de economía jurídiconormativa apalancada por un sistema informal de control y sanción; Precios Justos implicará la pretensión de control absoluto de la toda la economía, así como la movilización de todo el aparato estatal en el marco de una "Guerra Económica". Por su parte, el Sistema de Subsidios Egipcio suponen un equilibrio siempre delicado entre gasto público y contrato social, con una fuerte dependencia del sector externo; mientras que el Programa de Asistencia Nutricional Suplementaria constituiría un esfuerzo indirecto de control de precios, con una fuerte preocupación por la eficiencia en la distribución de recursos e identificación de la población más necesitada y una articulación con actores del mercado y agencia subnacionales, como garantía para la distribución de los beneficios. Volveremos sobre esto.

\section{Proposiciones}

Del proceso de inmersión en campo, proponemos identificar en este apartado algunos hallazgos preliminares, bajo la forma de cinco proposiciones.

Proposición \#1.- Existe un desborde de lo social, ahí donde sólo parecía haber un fenómeno económico de mercado.

Las políticas de control de precio nos enfrentan al diseño de instrumentos (políticos) para la administración de los flujos de relaciones que se nos presentan (en apariencia) de corte eminentemente económico. Sin embargo, se trata de arreglos institucionales que buscan facilitar no sólo el acceso a bienes de consumo básicos, sino también a partir de ellos regular una serie prácticas que exceden lo económico.

En el caso de Precios Cuidados, por ejemplo, las agencias estatales y el empresariado del retail local se involucraron en un proceso de definición/redefinición de sus relaciones, en donde el Estado se beneficiaría de la experiencia y recursos del sector privado para la puesta en marcha del programa, así como la extensión de sus capacidades como fruto de aceptación del programa, a cambio de la institucionalización de mesas de negociación, de una mayor cuota de importación de juguetes, textiles y electrodomésticos, y una creciente participación de mercado, en detrimento del denominado "canal tradicional" ${ }^{5}$.

Por el contrario, la versión venezolana de Precios Justos no supone negociación/renegociación de la relación entre el empresariado local y las agencias estatales, sino que -por el contrario- muestra un esfuerzo en la construcción del Estado y su autonomía como el único locus de realización de los intereses de la sociedad.

El caso de Egipto, por otro lado, nos pone frente a una economía moral, según la cual el plan debe ser accesible a todos. Una moralidad en donde seguridad alimentaria, protesta, pacto social, bienestar y factores internacionales, como la inserción en flujos internacionales de comercio y el beneficio de un "dividendo de paz" (Frerichs,2016) en la forma de

\footnotetext{
${ }^{5}$ En términos comparativos, en 2014 la facturación de los supermercados en Argentina creció un 36,2\% en comparación con el año anterior, según datos de Nielsen, con una tasa de crecimiento de $33,4 \%$. Por su parte, en el mismo período, los supermercados de origen chino registraron una caída de $8 \%$. En palabras de directivos de CASRECH: .En un radio de 400 metros a la redonda de una boca de cercanía de propiedad de una cadena de supermercado, los almacenes de barrio han perdido 7 puntos en sus ventas".
} 
asistencia de y vínculos intensos de comercio con las potencias occidentales, se implican unas a otras en un eco de referencias permanente.

En una palabra, el precio de los alimentos de consumo (y las pretensiones de regulación del acceso a los mismos), nos pone frente a un debate que va más allá de las cuestiones de corte económico. En definitiva, lo que se nos presenta en la superficie como un problema relativo al flujo de relaciones económicas, nos expone -en otro nivel- a cuestiones de orden político, como la constitución de alianzas de clase como fruto de la exportación de bienes-salarios y la necesidad de balancear el déficit fiscal (O'Donnell, 1977); a dimensiones sociológicas, como las cuestiones relativas a los sistemas de producción y distribución de los alimentos; y dimensiones antropológicas, en vistas a que no se trata sólo de la posibilidad de acceder a bienes de consumo para reproducir la fuerza de trabajo y saciar el hambre, sino que involucra sistemas de valores, hábitos y costumbres que hacen a la identidad de los pueblos..

La discusión sobre el valor de los bienes de consumo -y su control- se inserta al interior de este denso entramado de relaciones y abordajes. El sistema de precios deviene, en este sentido, en un punto de pasaje de relaciones sociales que exceden a un fenómeno funcional (económico) de superficie (una presunta mecánica semiótica del valor), para ponernos ante un dispositivo de regulación de un conjunto amplio de relación sociales, cuya intervención ha sido fundamental en diversas estrategias de desarrollo (Amsden, 1992) e -incluso- constituye -con mayor o menor fuerza, según el país- parte central del contrato social (Patel y McMichael, 2009).

Proposición \#2.- Los arreglos institucionales se erigen sobre la base de una tensión entre una economía moral del valor justo y una economía política del precio de mercado.

Los alimentos mantienen un estatuto ambiguo que define su doble condición de bienes de consumo y de símbolos elementales del pacto social. Es el entramado institucional que cada sociedad pone en juego lo que carga el peso sobre el mercado como mecanismo de regulación de esa tensión, sobre la política, a partir de mecanismos de provisión o sistemas de protección social, o bien sobre los individuos y las redes informales de familiarización.

Cada uno de los arreglos institucionales bajo análisis son la cristalización de una elección con miras a resolver esa tensión: qué parte de los riesgos se colec- tivizan y qué parte se mantiene en la esfera privada, sea ésta familiar o mercantil; qué parte corren por cuenta del Estado; y qué parte se deja librado a las fuerzas del mercado y los agentes individuales (Filgueira, 2013:19).

El caso venezolano, por ejemplo, se estructura sobre la base de la sanción de la Ley Orgánica de Precios Justos, la cual - en su articulado- manifiesta una serie de objetivos de profundos imperativos morales: "(...) asegurar el desarrollo armónico, justo, equitativo, productivo y soberano de la economía nacional, a través de la determinación de precios justos de bienes y servicios (...) a fin de proteger los ingresos de todas las ciudadanas y ciudadanos, y muy especialmente el salario de las trabajadoras y los trabajadores, el acceso de las personas a los bienes y servicios para la satisfacción de las necesidades".

En Egipto, en cambio, la subvención a productos preferencialmente consumidos por sectores sociales vulnerables, como el pan baladi, pone en evidencia una economía moral sustentada en una serie de reconocimientos legales efectivos de acceso al mismo, en el contexto de una serie de reformas que supusieron una pérdida de derechos, desde la liberalización de las tierras hasta la revisión del sistema de subsidios. Una economía moral del pan que tiene como premisa el derecho a la comida y condiciones justas y sustentables para su consumo. No en vano, la palabra egipcia para designar "pan" ("aish") también designa "vida".

Por otro lado, esta tensión se verifica también en el tiempo. Tiene bases y fundamentos históricos. Es un elemento siempre presente y siempre pendiente de resolución: la frontera en la cual se divide socialmente el trabajo del Estado, el mercado y la familia, es permanentemente negociada y definida/redefinida. Basta, en este sentido, mencionar las referencias de Adam Smith al hecho que en años de escasez parte de la sociedad atribuía su angustia a la avaricia de los comerciantes de maíz, quienes se convertían en el objeto de su odio e indignación (1776:526). Un odio que terminaba materializándose en leyes en contra de la compra y acopio de inventario.

Polanyi mismo, por otro lado, reconstruyó el doble proceso por el cual, mientras los mercado se expandían y crecía el volumen de bienes comercializados, en paralelo una red de instituciones y políticas era creada para controlar el accionar del mercado. En sus términos, "la sociedad se protegió a sí misma contra los peligros inherentes al sistema de mercado autorregulado. Ese fue el rasgo indiscutible de la 
historia de esa época" (Polanyi, 2017: 137).

Un "modelo paternalista" donde molineros y panaderos eran considerados sirvientes de sus comunidades que trabajan no por una ganancia, sino por una pago justo (Thompson, 1971:83), a la par que los nuevos procedimientos y prácticas de mercado eran cuestionadas. Thompson sostenía, a partir de una serie de registros históricos, que este modelo paternalista tenía una existencia ideal, pero también fragmentariamente real: era puesto en suspenso selectivamente- en años de buena cosecha y precios moderados, para ser revivido en momentos de turbulencia.

Nada parece haber cambiado sustancialmente, en algunas sociedades, en el devenir del debate en el tiempo, desde el siglo XVII a esta parte. Por el contrario, cada coyuntura solo parece actualizar (de manera particular, ajustada al tono de sus tiempos) sus formas. Una versión teatralizada, apenas actualizada en sus pliegues, de dramas ancestrales, siempre por concluir. Siempre por reiniciar.

Proposición \#3.- La tensión identificada en la proposición anterior se traduce en una amenaza siempre latente de acción colectiva.

Una práctica que también tiene raíces históricas profundas y que pasó a formar parte del repertorio -siempre disponible en la memoria y tradición popular- de acción colectiva. Una práctica ambigua, entre el beneficio individual y la protesta social (Serulnikov, 2017:155).

En lo que hace a nuestra investigación, no importa (a los efectos de la misma) la forma particular que tome la acción colectiva, sino la existencia y verificación empírica misma de esa amenaza. Su valor, en suma, radica en el momento mismo en que existe y es reconocida como potencialidad efectiva.

En Egipto, por ejemplo, desde la revolución de enero de 2011 el liderazgo político se ha vuelto cada vez mas contingente del poder de las calles (Frerichs, 2016). Sobre todo, a la luz de la memoria emotiva de los sectores populares. Son recurrentes, en esta línea, las referencias constantes a las protestas de 1977 en donde, como parte de una serie de compromisos con el FMI, el entonces presidente Sadat inició un proceso de reducción de subsidios que contemplaba cambios en el precio del pan fino, azúcar, arroz, gas butano para cocinar, gasolina y cigarrillos. $\mathrm{El}$ anuncio derivó en protestas de entre 2.000 y 4.000 personas en el Cairo y marchas de más de 20.000 personas en Alejandría (Sachs, 2012:31), las cuales registraron 77 muertos en 2 días y -desde entoncesconstituirían un miedo siempre latente y presente en cualquier intento de reforma.

De hecho, durante la década de 1980, según Sachs (2012), se verificó una política de cooptación y de "represión permisiva", donde sólo las acciones más confrontativas eran enfrentadas por el Estado; mientras que a partir de 1990 se reducirían las libertades políticas como respuesta al aumento de la violencia (sumado a una mayoría parlamentaria creciente, aumento de violencia extremista y control de los sindicatos).

En Argentina, en cambio, se evidenciaba una creciente expectativa - a nivel de la opinión públicade intervención del mercado de bienes de consumo masivo, así como descontento frente al aumento de la inflación. En este sentido, a principios de 2014, Argentina afrontaría un escenario de devaluación, y consecuente pérdida del poder adquisitivo, del orden del $40 \%$, a la vez que los índices de inflación registrarían la mayor alza mensual del período al alcanzar un $4.5 \%$ en enero. De hecho, el tema progresivamente devendría en un asunto de alta sensibilidad para la opinión pública (llegando en abril de 2013 a ser indicado como el segundo principal problema del país por el $47 \%$ de los consultados en un estudio de la consultora $\operatorname{IPSOS}^{6}$ ), hasta alcanzar en diciembre de mencionado año un crecimiento de las expectativas negativas sobre la situación económica personal y del país ${ }^{7}$.

Ahora bien, esta proposición encierra una paradoja: en la amenaza de violencia colectiva radica la mayor fuente de legitimidad a la intervención estatal y del desarrollo de sus capacidades. En Argentina, por ejemplo, según el citado estudio de IPSOS, más del $50 \%$ de la población encuestada se encontraba a favor que el gobierno controle los precios de los alimentos básicos y servicios. Incluso, el $43 \%$ declaraba que las empresas de consumo masivo deberían

\footnotetext{
${ }^{6}$ Conforme a los datos provistos por la Ficha Técnica del estudio en cuestión, se trata de una investigación cuantitativa, con un cuestionario semi estructurado compuesto por preguntas abiertas, con una duración aproximada de $25 \mathrm{mi}-$ nutos. La muestra total fue de 998 casos, tomada en todo el país entre hombres y mujeres, entre 16 y 75 años de todos los NSE, con un método de muestreo por cuotas de sexo, edad, nivel socio económico y región. Por último, el error muestral se calculó en $+/-3.09 \%$ y un nivel de confianza del $95 \%$.

${ }^{7}$ Según el mencionado estudio de IPSOS- Mora y Araujo, en mayo de 2012 un $25 \%$ de los encuestados manifestaba creer que el país estaría en peor situación dentro de un año y un $14 \%$ lo creía de su situación personal. Sin embargo, para diciembre de 2013, esos valores pasarían a representar el $34 \%$ y el $20 \%$, respectivamente.
} 
estar bajo directa administración del Estado.

Proposición \#4.- La tensión identificada en la $\mathrm{P} \# 2$, así como la amenaza latente de la $\mathrm{P} \# 3$, derivan en precedencia del Estado por sobre otros órdenes, así como un rol activo de sus agencias en el diseño de un sistema institucional que pueda establecer un punto de equilibrio entre tensiones y amenazas.

Esta proposición supone dos reconocimientos simultáneos. En primer lugar, que las crisis alimentarias no ocurren - simplemente- como fruto de fallas en las cosechas, sino también como fruto del acceso/no-acceso a provisiones estatales, redes de apoyo familiar, mercados de trabajo, entre otros. Es decir, son el resultado del concurso de una serie de decisiones que mencionábamos más arriba en relación a qué parte de los riesgos se colectivizan y qué parte se mantiene en la esfera privada, sea ésta familiar o mercantil; qué parte corren por cuenta del Estado; y qué parte se deja librado a las fuerzas del mercado y los agentes individuales.

Sin embargo, en segundo lugar, supone que la definición/redefinición de las fronteras entre Estado, familia y mercado como órbitas de satisfacción del bienestar siempre implica un saldo en favor del primero: un "dividendo estatal". En otros términos, nunca la mercantilización y/o la familiarización se presentan de forma absoluta. Por el contrario, ambos órdenes funcionan a partir de cierta arquitectura estatal que les sirve de base y le permite al Estado, a su vez, ampliar sus capacidades como fruto de esa articulación ${ }^{8}$.

El sistema de precios es un ejemplo contraintuitivo en este sentido. Aun cuando su funcionamiento se nos presenta como eminentemente económico, su uso -como sostenía Coase en 1932- supone un costo: los precios tienen que ser descubiertos, se deben desollar negociaciones, inspecciones, contratos y arreglos para resolver disputas. Un "costo de transacción" que no sólo realza la importancia del derecho en la discusión económica, sino que denuncia que pese al interés

\footnotetext{
${ }^{8}$ En esta línea, por ejemplo, Amengual documenta que, a propósito de las capacidades del Estado para controlar las violaciones a las normas laborales en los talleres de confección de ropa en Buenos Aires, este tipo de prácticas articulatorias entre el Estado y organizaciones de la sociedad civil, nos ponen frente a la necesidad de reconocer el rol que cumplen estos vínculos en la construcción de la capacidad del Estado (Amengual,2013:309). En términos de Scokpol, "la implementación de políticas adquiere su forma no sólo por los instrumentos políticos a disposición del Estado, sino también por el apoyo organizado que este pueda recibir de grupos sociales claves" (1985:20).
}

de los economistas en el sistema de precios, no se le prestó suficiente atención a los arreglos institucionales que gobiernan el intercambio (1994:6). Por el contrario, las empresas y el mercado se presentarían en nombre, pero no en sustancia.

En Argentina, por ejemplo, el Estado se vale de redes informales de la sociedad civil para ampliar el stock disponible de recursos destinados al control del programa. Esta articulación tuvo dos efectos directos. En primer lugar, posibilitó un crecimiento de la capacidad para penetrar en la sociedad civil y controlar que los productos definidos estén en las góndolas, al precio acordado y con la señalización especial definida. Según datos de una de las principales agrupaciones políticas participantes, en el inicio del programa se distribuyeron más de 500.000 panfletos con los listados de los precios y productos incluidos y participaron -solo de esa agrupación- 1.700 militantes, 400 en la Capital Federal y 1.300 en diferentes puntos del territorio bonaerense. En esta línea, en la Ciudad de Buenos Aires se instalaron frente a cadenas minoristas, como supermercados y autoservicios, 460 mesas de difusión, mientras que en la provincia fueron cerca 360 en distintos puntos.

Esta articulación posibilitó una ampliación del margen de la capacidad de control de los sólo 41 "relevadores" oficiales que disponía la Secretaría de Comercio, asegurando la capacidad de inspección, la cual -de lo contrario- no hubiese alcanzado para cubrir el total implicado de 2.000 bocas de expendio.

En segundo lugar, esta articulación con actores no estatales, posibilitó el establecimiento de un régimen de sanciones "no oficiales" vinculadas con cierto accionar ilegal, como el daño a la propiedad privada a partir de pintadas y "escraches", todas llevadas a cabo por organizaciones sociales y/o políticas.

Es decir, este tipo de articulación amplió -según nuestra primer investigación (Quiroga,2015)- los recursos estatales de control, monitoreo y sanción, a partir de cierto régimen de "estatalidad híbrida" (Dewey,2012): una forma particular de accionar del Estado que contempla el hecho que pueda actuar incluso de forma ilegal, a partir de agrupaciones sociales y políticas en este caso, para responder a demandas sociales. En otros términos - como sostiene el autor-, nuestro entendimiento de la "estatidad" no debe restringirse a la posibilidad de un Estado de proveer legalmente productos y servicios, sino también a la posibilidad de actuar incluso de forma ilegal para mejorar su eficacia (Quiroga,2015). Desde esta perspectiva, ni el poder del Estado, ni su capaci- 
dad, pueden entenderse en un sentido puramente legal. De hecho, las prácticas ilegales forman parte del stock de recursos usados para proveer seguridad a la población. Incluso, varios autores rescatan el hecho que la configuración historiográfica misma del Estado tiene en sus orígenes mismos una frontera difusa entre legitimidad e ilegitimidad de acción: "la mejor fuente de apoyo de un rey veces se encontraba en el mundo de la ilegalidad [outlaws]. La conversión de Robin Hood a aquero del rey puede ser un mito, pero uno que registra una práctica" (Tilly, 1985:173) ${ }^{9}$

La convocatoria de Precios Justos en Venezuela a inscribirse como "inspector popular" en el marco del denominado "Combate en la Guerra Económica" debe interpretarse en el mismo sentido, aun cuando (como adelantábamos), el Estado movilizó todos sus recursos al control del programa.

Por otro lado, en Egipto también se verificó una articulación del mismo tipo (si bien de corta duración: en enero de 2013, con la asunción de Bassem Ouda como Ministro de Abastecimiento y Comercio Interno, se activó un sistema de distribución de alcance nacional a través de unidades locales y asociaciones civiles, usando estructuras partidarias del Partico de la Libertad de la Hermandad Musulmán.

De esta forma, el stock de recursos punitorios disponibles al alcance del Estado, fundamentalmente inspecciones, multas y clausuras, se ven complementadas con instancias informales.

Se verifica, entonces, una primacía del orden estatal en la regulación las relaciones de mercantilización y familiarización. Después de todo, "(...) la creación de la riqueza no es ya solamente una función de la naturaleza o del mercado; sino que implica también un estructura estatal efectiva" (Evans, 1995:6), en sociedades donde - cada vez más- el Estado debe garantizar, a su vez, niveles aceptables de bienestar.

En una palabra, los mercados no son la emergente de un curso "natural" de las cosas, sino un fenómeno político en donde el Estado cumple una función decisiva. En este punto, la intervención estatal no constituye -simplemente- un esfuerzo por proteger a la sociedad del mercado, sino también una fuerza clave el desarrollo de este último y de la mercantilización de la vida social.

\footnotetext{
${ }^{9}$ La traducción es nuestra. Esta línea historiográfica de investigación sobre las características, funciones del Estado moderno, así como su constitución, a partir del desarrollo de capacidades extractivas, de acumulación de capital, y bélicas y de ejercicio de la violencia legítima, es profundizada por autores como: Lane (1958) y Herbst (1990).
}

Sin embargo, por estar en definición y redefinición permanente, la primacía del orden estatal no anula la posibilidad de su resistencia, sino todo lo contrario. En Egipto, por ejemplo, las reformas sancionadas en el sistema de subsidios en 2014 suponen un intento por (re)mercantilizar ciertas relaciones. En este sentido, por ejemplo, se dejó de subsidiar la harina que se distribuía a las panaderías. De esta forma, las mismas la obtendrían de los molinos a precio de mercado y venderían el pan al precio fijado. El Gobierno, por su parte, subsidiaría la diferencia de forma que este nuevo esquema debería permitir controlar las fugas al mercado negro, a la vez que las panaderías se convierten en competidores de mercado con nuevos incentivos para mejorar su eficiencia y productividad para obtener una mejor ganancia. Este nuevo esquema, según las fuentes relevadas, habría posibilitado bajar las denuncias por bajo peso de las rodajas: de 37.339 a 45.598 incidentes en 2013 a 11.860 a 14.102 en 2015 .

De esta forma, aun cuando le sirva de sustento al resto, el orden estatal es permanente renegociado en sus límites. Es más, lejos del lugar de subordinación que la literatura le asigna al empresariado, el mismo juega un papel central en este entramado de tensiones (Quiroga, 2017a), en el marco de lo que algunas han denominado un "régimen corporativo de alimentos" (Patel y McMichael,2009), que tienen en el Estado -sus incentivos, sistemas de titulación de derechos e intervenciones en el tejido social- su punto de articulación.

Proposición \#5.- La forma particular del diseño institucional lleva sobre sí las marcas de un sistema estructurado y jerarquizado de determinaciones.

Ahora bien, el accionar del Estado y sus agencias, derivado de la proposición 4 no puede surgir de cualquier manera. Por el contrario, se encuentra condicionado (limitado y posibilitado a su vez) por un sistema de determinaciones que definen la particularidad de su forma.

Eso supone -en primer lugar- partir de una concepción no-ideal, donde para ser captadas en la especificidad de su rol, las agencias estatales deben ser analizadas en la materialidad misma de su realidad empírica, en donde pueda relevarse su configuración particular, en tanto que producto histórico de confrontaciones y disputas, así como la articulación efectiva que nos ofrece en términos de estructuras, normas, comportamientos y recursos humanos, materiales y tecnológicos (Oszlak y O’Donnell, 1995). 
Sin embargo, para entender la particularidad de su forma, debemos partir en el análisis por identificar y describir la configuración particular de operadores que nos ofrece en su superficie (sus características), para luego reconstruir la relación entre éstos y el sistema de determinaciones (estructurado en diversos niveles de abstracción) que le sirven de condición de producción y condicionan/posibilitan la forma efectiva que esas agencias (y las relaciones que proponen) asumen.

Un esfuerzo de este tipo (aun cuando excede los objetivos del presente trabajo) nos llevaría a encontrarnos, en el nivel superior de tipificación lógica (el de mayor abstracción) con las restricciones, condicionamientos y posibilidades propias del régimen político, en su doble condición de reglas de acceso al poder y ejercicio del mismo (Mazzuca,2012 y 2002).

En caso de Estados Unidos, por ejemplo, la estructura de organización burocrática puesta en juego, lleva impresa sobre sí los límites mismos del régimen político que le da origen, como consecuencia de un déficit histórico del Estado nacional norteamericano, en lo que algunos han definido como federalismo descentralizado, débil y fragmentado (Skocpol, 1995:138). En este sentido, si bien los criterios de elegibilidad del programa se definen a nivel federal, lo que garantizaría cierta uniformidad de criterio, son los Estados subnacionales quienes definen el punto en la escala de rangos de ingresos, tamaño y composición del hogar, bienes, gastos médicos, estatus inmigratorio o de ciudadanía, entre otros.

De la reconstrucción analítica del esquema general del programa se desprende una estructura del tipo híbrido en la tipología de Mintzberg (2003). Incluso, del tipo particular que el mismo autor reconoce bajo el nombre de "burocracia de copia de carbón" (2003:268) en donde características de la Forma Divisional y la Burocracia Mecánica se cristalizan en una estructura que reconoce divisiones (en nuestro caso geográfico, materializado en 7 oficinas regionales), pero las cuales son exactamente iguales entre sí. No se manifiestan diferencias (en sus actividades y modalidades de relacionamiento con los diversos actores en territorio) como respuesta a las particularidades de los "mercados" regionales que atiende; a la vez que el "cuartel general" (por mantener la terminología del autor) concentra para sí determinadas funciones críticas: mientras la Casa Central mantiene control sobre todo lo relativo a la legislación, la sanción de regulaciones, el desarrollo de la política a nivel nacional y la definición del contenido de todas las comunicaciones; las oficinas regionales se encargan de implementar las regulaciones, asistir técnicamente, supervisar el programa y su ejecución, y realizar las evaluaciones.

Esto último pone en evidencia que si bien las divisiones son -efectivamente- creadas en línea con los mercados atendidos y se les brinda ciertas funciones operativas que necesitan para realizar sus tareas en esos mercados, no asumen el atributo de autonomía propio de las divisiones bajo la Forma Divisional (pura). De esta forma, la articulación entre atributos (predominantes) de la Burocracia Mecánica y otros (incipientes) de la Forma Divisional, no radica -a nuestro entender, al menos-, como sostiene Mintzberg, en un efecto de la evolución lógica de la forma Burocrática Mecánica, que -como consecuencia de la diversificación de servicios para atender distintos mercados- transitaría a una Forma Divisional; sino que -por el contrario- el origen de este híbrido radica en cuestiones contradictorias entre sí, como son las necesidades operativas de ganar capilaridad, garantizar ciertos grados de autonomía y libertad a los estados subnacionales, a la vez que controlar la ejecución de los fondos.

En suma, esta articulación se estructura sobre una imposibilidad: el servicio civil estadounidense carece de estructuras que puedan llegar directamente a las localidades, por lo que sólo puede contentarse con una combinación de incentivos financieros y recursos legislativos para que las cosas se hagan. En términos de Skocpol, "(...) el Gobierno Federal en parte soborna y en parte da órdenes [bosses] a los Estados subnacionales y locales, así como a los grupos no-gubernamentales; posibilitando que lo ayuden a hacer lo que él no podría hacer por sí mismo" (1995:9). Es la red de comercialización y el aparato burocrático subnacional los que dotan al programa de la infraestructura material sobre la cual éste puede actuar. Sobre todo, a la luz del déficit histórico del Estado Federal en este sentido ${ }^{10}$, siendo caracterizado por varios autores como un federalismo descentralizado, débil y fragmentado (Skocpol, 1995:138).

En un segundo nivel, tendríamos los condicionamientos y posibilidades propias del patrón de acu-

\footnotetext{
${ }^{10}$ La organización federal se estructura sobre una débil red de Cortes y partidos políticos (Skocpol, 1995), las cuales actuarían como organizaciones clave en la constitución de la estatalidad de orden nacional, a lo largo del país. Precisamente por la división federal que existe a su interior, son los procedimientos partidarios los que habrían dotado de coherencia a una serie de instituciones desarticuladas del aparato gubernamental, mientras las Cortes determinarían el sentido y el efecto de la Ley misma en todo el territorio (1995:21).
} 
mulación, en tanto que forma de producción, apropiación, distribución y utilización del excedente en una sociedad determinada y la institucionalidad política que le da sustento. Este punto es la consecuencia lógica de entender al Estado no sólo como un agregado heterogéneo de agencias de gobierno, con distribuciones diferenciales entre las mismas de recursos y capacidades para la acción, sino también como un actor clave en lo económico, tanto en lo relativo al proceso de acumulación de capital, como de transformación del perfil productivo de un país y la redistribución del ingreso (Evans y Rueschemeyer, 1985).

El caso de Egipto, por ejemplo, la articulación propuesta por el programa evidencia, de forma significativa, restricciones y posibilidades relativas a este nivel, en vistas a la relación dinamiza que el programa mantiene con los procesos de liberalización de la agricultura, los planes de ajuste y reforma del Estado, la participación en los flujos de asistencia internacional y la presión sobre el gasto público. El sistema de desabastecimiento local y abastecimiento internacional es beneficiario del proceso de liberalización de la agricultura entre 1986 y 1992, sobre la base del objetivo declarado de "activar las fuerzas de mercado en la determinación del valor de renta de la tierra (...) mejorando de esa forma la eficiencia en la distribución de la renta" (Frerichs,2016:16).

Esto habría afectado, según estimaciones, a más de 900.000 productores (cerca del $25 \%$ ) quienes quedaron despojados de sus tierras (Frerichs,2016:17), a la vez que forzaron a una creciente importación de productos, así como la inclusión de Egipto en programas de asistencia alimentaria, como "Food for Peace", a partir del cual productos agrícolas producidos en Estados Unidos eran vendidos a Egipto sobre la base de préstamos subsidiaros desde inicios de 1970 hasta los primeros años de la década del 1990, cuando su desempeño fiscal mejoró como fruto de la condonación de su deuda tras la Guerra del Golfo.

Algo similar puede decirse en relación el SNAP en Estados Unidos: el mismo favoreció el proceso de distribución privada, donde la articulación con las cadenas de comercialización posibilitó un mayor alcance en la distribución de los beneficios, a través de una tarjeta electrónica, la cual puede ser usada sólo en negocios autorizados. Al día de hoy, la cantidad de tiendas autorizadas asciende a 261.000, $80 \%$ de los cuales son supermercados o hipermercados (CBPP, 2016). Al punto que esta articulación dinamizaría el ciclo económico: el programa es considerado por muchos economistas, en este sentido, como un ele- mento central en el estímulo de la actividad económica. Según Moody's cada dólar distribuido entre los beneficiarios del programa, se generan $\$ 1,70$ en actividad económica (Rosenbaum, 2013:4).

En tercer lugar, estarían las limitaciones/posibilidades relativas a las estructuras organizacionales y procesos de la agencia en cuestión y su memoria institucional. Se trata del nivel menos cuestionado en la literatura y siempre presente como "entrada" clásica al análisis de las organizaciones. El mismo se componen no sólo de recursos materiales, financieros, humanos y tecnológicos, sino también de principios, normas y rutinas que regulan el accionar y de estructuras con grados diversos de diferenciación estructural, especialización funcional e interdependencia (Oszlak y O’Donnell, 1995).

El caso argentino, por ejemplo, en el período comprendido entre 1967 y 1989, los controles de precio serían la norma en Argentina, mientras que los períodos de "libertad de fuerzas de mercado" quedarían limitados a 30 meses, sobre un total de 274 meses (Fiel, 1990). En una palabra, la "solución" de los controles estaba disponible -a la vez que constituía una parte de la "memoria viva" de las agencias de gobierno implicadas- al momento de identificarse el problema y verificarse las condiciones de posibilidad necesarias. Soluciones pasadas, en suma, quedan como opciones disponibles a ser actualizadas, redefinidas, negociadas o descartadas a futuro. Un punto que ilustra que no existe prioridad ni lógica ni ontológica de los problemas, por sobre las soluciones.

En segundo lugar, la novedad del programa radica en el desvío que sus arreglos y expresiones organizacionales supuso de la estructura burocrática verificada en promedio en las agencias estatales nacionales. Sobre todo, en lo relativo a la expresión mínima formal en la que se cristaliza, una estructura de 41 relevadores a nivel nacional, y el apoyo en organizaciones sociales, a los efectos del control, monitoreo y sanción.

Incluso, el hecho que para hacer efectivo y capilar su aparato de control y monitoreo el Estado recurra a cierta hibridez, debe ser leído a la luz de cierta desconfianza de la política sobre el nivel de compromiso de las estructuras burocráticas para el cumplimiento de objetivos específicos, donde sólo la militancia de base ofrecía las condiciones necesarias en términos de verticalidad, conciencia de misión y fines, para el control del programa.

En este sentido, puede decirse que al evidenciar un 
desempeño por debajo de los 50 puntos porcentuales, tanto en los índices de mérito, capacidad funcional y consistencia estratégica (Zuvanic e Iacoviello, 2005; Echebarría y Cortázar, 2006), el sistema de servicio civil argentino presenta ciertas fugas y pérdidas entre las instancias de tomas de decisión y la ejecución misma de los programas. Se trata de un atributo clave que define, a nuestro entender, la necesidad de recurrir a grupos informales, por fuera de la estructura del Estado, para el cumplimiento de los objetivos.

Por último, en el nivel siguiente, tendríamos el orden relativo al arte de oficio, en los términos definidos por Lynn (2006); es decir, las habilidades y destrezas que posibilitan hacer de las rutinas burocráticas oportunidades en la generación de cambios organizaciones o en la construcción personal de los ciertos funcionarios.

Este nivel está íntimamente vinculado con los desarrollos de Moore en relación a el reconocimiento de la utilidad potencial de la innovación y creatividad de la burocracia, a partir del desarrollo de canales formales que permitan su expresión y control democrático (Moore, 1998). Se trata de un punto que torna aún más crítico el análisis y consideración de los denominados "patrones de gestión de políticas públicas" menos formalizados, tal y como los conceptualiza Andrenacci (2015): un campo de cuestiones emergentes en un zona de confluencia entre problemas estructurales de capacidad estatal (aspectos institucionales, operativos, de coordinación o administrativos, medios financieros, cultura organizacional, capacidad y capacitación de los recursos humanos) $\mathrm{y}$ aspectos contingentes que derivan de las capacidades y atributos individuales de actores singulares, como funcionarios.

En el caso de Estados Unidos, por ejemplo, es al nivel de las Oficinas Regionales y en articulación con los Estados subnacionales y las cadenas de retail local, en donde se presentan algunas prácticas (no formales) que pueden afectar la suerte del programa. Algunos trabajos (Gabor et al, 2003) han señalado que es este nivel en donde puede verificarse algunas prácticas que afectarían la decisión de aplicar o no al beneficio; entre ellas: (a) la disponibilidad de información sobre el programa, así como campañas de difusión; (b) la disponibilidad de formularios de aplicación, en lugar de tener que realizar alguna entrevista como precondición para poder acceder al material (como ocurre en el $10 \%$ de los casos); (c) la accesibilidad de las oficinas, sobre todo en lo que hace a la atención en horario extendido (lo que posibilita que la gente se acerque después de su trabajo), así como la atención en lugares con acceso de transporte público y el pago de los costos de movilidad, a través de vouchers o viajes directos a la oficina; y (d) prácticas que pueden ayudar a grupos con vulnerabilidad a atravesar el proceso, como la disponibilidad de traductores para aplicantes que no hablen inglés.

\section{Consideraciones finales}

En los apartados anteriores hemos intentado delinear un esbozo -impreciso en sus márgenes- de un proceso por definición incompleto. Un juego de relaciones y tensiones, condensadas en torno a los sistemas de precios y los regímenes de acceso a los alimentos, que si reviste interés para la investigación lo hace porque pone en juego -en última instanciael pacto social como fruto de una tensión entre una economía moral del valor justo y una economía política del precio de mercado. Un pacto en definición/redefinición permanente, donde el Estado y su arquitectura mantienen, según nuestra formulación, cierta primacía por sobre las pretensiones de mercantilización y familiarización -y su locus de satisfacción: el mercado y la sociedad civil, respectivamente, aun cuando esa precedencia sea siempre discutida; siempre incompleta.

He ahí el punto central de nuestra investigación: en mercados de transiciones incompletas entre economías morales de provisión y economías políticas de mercado, las políticas de control de precios son parte integral del pacto social. Un pacto que solo se podría sostener a costa de las pretensiones de mercantilización y familiarización absoluta de la vida social.

En definitiva, es esta tensión e inestabilidad, materializada en amenazas latentes de acción colectiva, no sólo la condición misma de la prioridad del orden estatal, sino también la que hace de las capacidades estatales un elemento central de análisis. Si existe precedencia de las relaciones estatales, entonces la problematización del Estado y sus capacidades devienen en una vía de acceso preferencial para su estudio.

En este orden de ideas, y aun cuando es cierto que el enfoque sobre el Estado "(...) debe ser conju$\operatorname{gad}[\mathrm{o}]$ con elementos que permitan abordar el otro extremo de la relación: los actores empresarios y sus formas de organización y acción" (Castellani y Llampart,2012:161), se trata de reconocer el hecho 
que -por su función en el proceso de acumulación, la dependencia del aparato estatal con respecto a la misma y el conjunto de relaciones que regula y permea- en algún punto, el análisis de las estructuras estatales no sólo deben ponerse en relación con el análisis de las estructuras de mercado (Evans y Rueschemeyer,1985), sino que son su punto de partida necesario.

Por otro lado, no cualquier conceptualización de la noción de capacidad estatal deviene en un instrumento útil, en este sentido. En lo que a nosotros respecta, hemos intentado dar cuenta de una alternativa, a partir de una operacionalización en base a dimensiones observables, que permite iluminar no sólo la organización al interior del sujeto estatal, sino incluso la relación con su otredad (los vínculos formales e informales con actores no-estatales, la legitimidad que gozan sus intervenciones para con el resto del cuerpo social, y su capital de acción interinstitucional), así como la reconstrucción del sistema de determinaciones que condiciona la forma misma con la emerge en la superficie de la trama social.

Esto nos deja frente a una paradoja de interés: en el mismo movimiento por el cual la discusión sobre el valor de los bienes de consumo nos pone al interior de un entramado denso de relaciones y abordajes que exceden al hecho económico y a cierta predominancia de lo estatal, asistimos y quedamos expuestos a los límites de la capacidad absoluta del Estado y sus agencias para intervenir el tejido social de forma coordinada y consistente. Cada vez más, su realización, la de sus políticas y fines, dependen de la articulación con actores no-estatales, de prácticas informales y recursos ilegales.

De esta forma, siempre en definición y redefinición permanente, la precedencia de lo estatal no anularía la posibilidad de su resistencia, sino todo lo contrario. El orden estatal es permanentemente renegociado en sus límites por diversos actores y las políticas de control de precios y acceso a los alimentos derivan en un campo de investigación privilegiado para entender la lucha por la definición/redefinición del pacto social y los vínculos entre Estado, mercado y sociedad.

Llegamos entonces, a la identificación con el síntoma: en el barro de la historia de las capacidades del Estado, es sus pliegues, en sus falencias, en su intervención de otros órdenes y arreglos de lo social, en su funcionamiento híbrido, al límite de la legalidad misma que sanciona, no encontramos un desvío o una anomalía, sino más bien, la verdad de su fun- cionamiento. O más aún: la condición de su eficacia.

\section{Referencias}

Amsden, A. (1992). Asia's Next Giant: South Korea And Late Industrialization. Oxford University Press, New York.

Bienen, H. S. y Gersovitz, M. (1986). Consumer subsidy cuts, violence, and political stability. Comparative Politics, 19(1):25-44.

CBPP (2016). Policy Basics: Introduction to SNAP. Center on Budget and Policy Priorities.

Clinard, M. B. (1969). The Black Market; A Study of White Collar Crime. New York Rinehart \& Company.

Esping-Andersen, G. (1993). Los tres mundos del Estado de Bienestar. Alfons el Magnànim,, Valencia.

Evans, P. (1995). Embedded Autonomy: States And Industrial Transformation. Princeton University Press, Princeton.

Evans, P. y Rueschemeyer, D. (1985). The sate and economic transformation: Toward an analysis of the conditions underlying effective intervention. En Evans, P., Rueschemeyer, D., y Skocpol, T., editores, Bringing the State Back, pp. 3-43. Cambridge University Press, New York.

Fiel (1990). Control de precios e inflación. La experiencia argentina reciente. Ediciones Mantieal, Buenos Aires.

Filgueira, F. (2001). Entre pared y espada: ciudadanía social en América Latina.

Filgueira, F. (2013). Los regímenes de bienestar en el ocaso de la modernización conservadora: posibilidades y límites de la ciudadanía social en américa latina. Revista Uruguaya de Ciencia Política, 22(2):17-46.

Filgueira, F. (2014). Hacia un modelo de protección social universal en América Latina, volumen 188. CEPAL. Serie Políticas Sociales, Santiago de Chile.

G., J. y Neeb, L. (1974). Confessions of a Price Controller. Dow Jones-Irwin, Homewood.

Galbraith, J. (1941). The selection and timing of price controls. The Review of Economic Statistics, 23(2):82-85. 
Isaacs, J. (2008). The costs of benefit delivery in the food stamp program. Electronic Report from the Economic Research Service, 39.

Martinez Franzoni, J. (2008). Welfare Regimes in Latin America: capturing constellations of Markets, Families, and Policies. Latin American Politics and Society. University of Miami.

Mesa-Lago, C. (2004). Evaluación de un cuarto de siglo de reformas estructurales de pensiones en américa latina. Revista de la CEPAL, 84.

Moore, M. H. (1998). Gestión Estratégica y Creación de Valor en el Sector Público. Paidos, Barcelona.

Ocampo, J. A. (2008). Las concepciones de la política social: universalismo versus focalización. Nueva Sociedad, 215.

Oszlak, O. y O'Donnell, G. (1995). Estado y politicas estatales en América Latina: hacia una estrategia de investigación.

O'Donnell, G. (1977). Estado y alianzas en la argentina, 1956-1976. Desarrollo Económico, 16(64):523-54.

Polanyi, K. (2017). La Gran Transformación. Crítica del liberalismo económico. Fondo de Cultura Económica, México.

Quiroga, J. (2015). Price control policies and state capacities. Discipline, transfer and informal networks of control, monitoring and punishment. The "Precios Cuidados" program in Argentina and its acceptance by local retailers.

Quiroga, J. P. (2017a). Crítica a la razón binaria: poder empresarial y agenda global en el marco de políticas de protección de mercado. el caso de precios cuidados en argentina. Revista Estudios de Políticas Públicas, 5(1):122-138.

Quiroga, J. P. (2017b). El síntoma de la estatalidad: los controles de precios y las capacidades estatales. el caso de precios cuidados en argentina. Revista Perspectivas de Políticas Públicas, $6(13)$.

Rockoff, H. (1984). Drastic Measures: A History of Wage and Price Controls in the United States. Cambridge University Press, New York.

Rosenbaum, D. (2013). SNAP Is Effective and Efficient. Center on Budget and Policy Priorities.
Sachs, R. (2012). On Bread and Circuses: Food Subsidy Reform and Popular Opposition in Egypt. Center for International Security and Cooperation, Stanford University.

Schuettinger, R. y Butler, E. (1978). Forty Centuries Of Wage And Price Controls: How Not to Fight Inflation. The Heritage Foundation, Washington, D.C.

Serulnikov, S. (2017). Pobreza y revuelta de subsistencia. los saqueos de 1989 en argentina. Historia Social, pp. 63-85.

Skocpol, T. (1995). Social Policy in the United States. Princeton University Press, Princeton.

Taussig, F. (1919). Price fixing as seen by a price-fixer. Quarterly Journal of Economics, $33(2): 205-241$.

Thompson, E. (1971). The moral economy of the english crowd in the eighteenth century. Past and Present, 50:76-136.

Tilly, C. (1985). War making and state making as organized crime. En Evans, P., Rueschemeyer, D., y Skocpol, T., editores, Bringing the State Back, pp. 3-43. Cambridge University Press, New York.

USDA (2016). Food and Nutrition Service. 2016 Explanatory Notes. The Office of Budget and Program Analysis.

Zuvanic, L. e Iacoviello, M. (2005). La burocracia en américa latina. ICAP-Revista Centroamericana de Administración Pública, 58-59:9-4. 